\title{
Multiplicative cascade processes and information integration for predictive mapping
}

\author{
Q. Cheng ${ }^{1,2}$ \\ ${ }^{1}$ State Key Lab of Geological Processes and Mineral Resources, China University of Geosciences, Beijing 100083, \\ Wuhan 430074, China \\ ${ }^{2}$ Department of Earth and Space Science and Engineering, Department of Geography, York University, Toronto, \\ M3J1P3, Canada
}

Correspondence to: Q. Cheng (qiuming@yorku.ca)

Received: 1 April 2010 - Revised: 5 December 2011 - Accepted: 22 December 2011 - Published: 11 January 2012

\begin{abstract}
This paper presents a new model proposed on the basis of multiplicative cascade process (MCP) theory for integrating spatial information to be used for mineral resources prediction and environmental impact assessment. Probability of a spatial point event is defined as the probability that a small map calculating unit (map unit) randomly selected from a study area contains one or more points. The probability that such unit randomly selected from a subarea with known spatial binary map patterns (evidential layers) contains one or more points is defined as the posterior point event probability. In this paper, processes of integrating multiple binary map patterns that divide the study area into smaller areas with updated posterior probabilities are viewed as multiplicative cascade processes resulting in a new log-linear model for calculating conditional probabilities from the multiple evidential input layers. The coefficients (weights) involved in this model measuring degree of spatial correlation between point event and the evidential layers are found to be associated with singularity indices involved in multifractal modeling. It is demonstrated that the model is simple and easy to be implemented in comparison with the existing weights of evidence model which is commonly applied in spatial decision modeling. In addition, the posterior probability as the end product of a multiplicative cascade process can be used to describe multifractality and singularity which are useful properties for characterizing spatial distribution of predicted point events. A case study of tin mineral potential mapping in the Gejiu mineral district in China is used to illustrate principles and use of the modeling process. Four binary layers: formation of limestone, buffer distance for intersections of three groups of faults, local and regional geochemical anomalies of elements $\mathrm{As}, \mathrm{Sn}, \mathrm{Cu}, \mathrm{Pb}, \mathrm{Zn}$ and $\mathrm{Cd}$, were combined for mapping potential areas for occurrence of tin mineral deposits.
\end{abstract}

\section{Introduction}

Singular physical, chemical and biological processes can result in anomalous energy release, mass accumulation or matter concentration that, generally, are all confined to narrow intervals in space or time (Cheng, 2007a). Singularity is a property of non-linear natural processes, examples of which include cloud formation (Schertzer and Lovejoy, 1987), rainfall (Veneziano, 2002), hurricanes (Sornette, 2004), flooding (Malamud et al., 1996; Cheng 2008), landslides (Malamud et al., 2004), forest fires (Malamud et al., 1996) and earthquakes (Turcotte, 1997; Cheng et al., 1994a). The end products of these non-linear processes can all be modeled as fractals or multifractals.

Hydrothermal processes are special types of singular process occurring in the Earth's crust; they can produce ore deposits characterized by high concentrations of metals and aggregate spatial distributions with fractal or multifractal properties (Mandelbrot, 1989; Carlson, 1991; Cheng et al., 1994b; Blenkinsop, 1994; Agterberg, 1995; Cheng and Agterberg 1996; Cheng, 2003; Raines, 2008; Hronsky, 2009). Hydrothermal mineral deposits also often exhibit non-linear features with respect to ore element and associated toxic element concentration values in rock and related surface media such as water, soil, stream sediment, till, humus and vegetation (Cheng et al., 1994a; Turcotte, 2002; Xie and Bao, 2004; Agterberg, 2007a; Xie et al., 2007; Cheng and Agterberg 2009; Ford and Blenkinsop, 2009; Hronsky, 2009). These properties can be used for delineating target areas for finding undiscovered mineral deposits in support of mineral exploration and mineral resources planning. For example, element concentration anomalies in various media are commonly identified by geochemical exploration techniques for recognition of target areas for predicting mineral deposits. Based on mineral deposit genetic models, specific 
geological features that control formation of mineral deposits can also be used to reduce the sizes of areas favorable for potential occurrences of undiscovered mineral deposits. For example, hydrothermal mineral deposits may occur around intrusive rocks; thus certain buffer distance around intrusions can be delineated as favorable area for occurrence of mineral deposits. Combining buffer zone around intrusions with other types of geological, geophysical and geochemical factors such as fault structures that control distribution of mineral deposits can further reduce the target areas for mineral exploration. This paper aims to demonstrate that the concepts of multiplicative cascade processes (MCP) and singularities as the end products of MCP can be applied to model the processes of combining multiple geo-variables (evidence) to map potential areas for discovering new mineral deposits. A new model is developed to associate the posterior probability of a unit area containing mineral deposits and conditions observed in the unit area as multiple attributes. Using this model, posterior probabilities can be calculated from the input conditions and their associations with mineral deposits. These posterior probabilities can be considered as resulting from multiplicative cascade processes that may depict multifractality and singularities which can be characterized by fractal and multifractal models.

\section{Multifractal model and singularity distribution}

In order to show the potential association between multiplicative cascade processes and information integration processes we first briefly introduce the concepts of multiplicative process, simple multifractal model and associated singularities. There are several formulisms for representing multifractals; for example, deterministic and stochastic models. More information about various multifractal models can be found in Schertzer et al. (1997). Complete review of various multifractal models is out of the scope of this paper. Here we will only present some assumptions and relevant mathematical notation for deterministic multifractals. Similar discussions can be partially applied to stochastic multifractal models. Assume a measure, $\mu$, defined in a small area of linear measuring size, $\varepsilon$ satisfies

$\mu(\varepsilon) \propto \varepsilon^{\alpha}$

where $\propto$ stands for "proportional to" and $\alpha$ is the singularity index, also known as the coarse Hölder exponent; $\mu$ is a function of scale $\varepsilon$ which possesses isotropic scale invariance property so that the following ratio of logarithmic transformations of $\mu$ and $\varepsilon$ gives scale independent index when $\varepsilon$ approaches zero

$\alpha \propto \frac{\log [\mu(\varepsilon)]}{\log \varepsilon}$

The values of $\alpha$ usually vary in a finite interval $\left[\alpha_{\min }, \alpha_{\max }\right]$ for deterministic multifractals, but for other models, for example, whole family of the Universal Multifractal models
(Schertzer and Lovejoy, 1987) that include both the $\beta$-model (Frisch et al., 1978) and the log-normal model (Yaglom, 1966), the bound of singularity might be infinite (Lovejoy and Schertzer, 2007). For some models the value of singularity can be negative and fractal dimension can be also negative. According to the distribution of the value of $\alpha$, the entire mapped area can be classified into subsets or fractals, each of which possesses different singularity value $\alpha_{i}$ and, accordingly, different fractal dimensions $\left(f\left(\alpha_{i}\right) \leq 2\right)$. This is the reason that the field of $\mu$ is described by the term "multifractality". The fractal dimension function $f(\alpha)$ and the singularity $\alpha$ can be estimated by various multifractal methods such as the method based on partition function (Halsey et al., 1986) and gliding box multifractal method (Cheng, 1999), just to name a few. For convenience of discussion, in this paper, we will use several terminologies pertaining to the multifractal model on the basis of partition function (Halsey et al., 1986), the entire study area can be partitioned into smaller subareas of equal size $\varepsilon \times \varepsilon$ and the measure of each such small area can be defined as $\mu(\varepsilon)$. Three functions: mass exponent function, $\tau(q)$, coarse Hölder exponent, $\alpha(q)$, and fractal spectrum function, $f(\alpha)$, can be introduced. They are associated according to the following relations (Halsey et al., 1986)

$$
\begin{aligned}
& \sum_{\alpha(q)=\tau^{\prime}(q)}\left[\varepsilon^{\tau(q)}\right. \\
& f(\alpha)=a q-\tau(q)
\end{aligned}
$$

where $q$ is the order of moment and the summation in the first equation is applied for all subareas of equal size $\varepsilon \times \varepsilon$ with positive measure $\mu$. From this formulation we can extract the following properties. When $q=0$ and $\alpha(0), f(\alpha(0))=$ $-\tau(0)$, reaching the maximum value of $f(\alpha)$ which corresponds to the box-counting fractal dimension. If the measure covers the entire 2-D set, then the box-counting dimension equals 2 , otherwise it is less than 2 ; when $q=$ 1 and $\alpha(1), f(\alpha(1))=\alpha(1)-\tau(1)$. If the first moment $\sum \mu(\varepsilon)=$ constant, then $\tau(1)=0$ and $f(\alpha(1))=\alpha(1)$. If we assume the number $N_{\alpha}(\varepsilon)$ of areas with size $\varepsilon \times \varepsilon$ covering the entire subset bearing the singularity $\alpha$, and the fractal dimension $f(\alpha)$ of this subset are related by

$N_{\alpha}(\varepsilon) \propto \varepsilon^{-f(\alpha)}$

The total measure of the subset can be expressed as

$N_{\alpha}(\varepsilon) \mu_{\alpha}(\varepsilon) \propto \varepsilon^{-f(\alpha)+\alpha}$

Since the total measure of the subset is less than the total measure of the entire set, the following relation must hold true:

$\alpha \geq f(\alpha)$

If relation (6) is not true, then the total measure on the subset $(5)$ would become infinity when $\varepsilon \rightarrow 0$. The frequency distribution of measure characterized by singularity $\alpha$ in the 
mapped area can be described by the fractal dimension spectrum function $f(\alpha)$; this function reaching its maximum value $f(\alpha(0))$ or the box-counting dimension of the support of $\mu$ at $\alpha(0)$. This implies that the majority of the area has measure characterized by $\alpha \approx \alpha(0)$, whereas areas with values $\alpha>\alpha(0)$ or $\alpha<(0)$ are more irregular and with fractal dimensions $f(\alpha)<f(\alpha(0))$. Since the relation (6) holds true for all $\alpha$ and $f(\alpha)$, the singular areas with enrichment of the measure due to $\alpha<2$ must have dimension $f(\alpha)<2$ and those areas with measure depletion due to $\alpha>2$ must have dimension $f(\alpha) \leq 2$; the equal sign applies only at $\alpha(0)(\geq 2)$.

\section{Multiplicative cascade processes and multifractal distributions}

The theory and concepts of multiplicative cascade processes play a fundamental role in explaining the generic consequence of scale invariant dynamics including turbulent intermittency and other non-linear processes (Schertzer and Lovejoy 1985, 2007; Schertzer et al., 1997). There are several types of cascade models such as the log-normal model (Yaglom, 1966), $\alpha$-model (Schertzer and Lovejoy, 1984) and p-model (Meneveau and Sreenivasan, 1987), to just name a few. A review of these models can be found in Schertzer et al. (1997). The model of de Wijs is a simple binomial multiplicative cascade model for generating log-normal distribution (de Wijs 1951). It became a multifractal model known as p-model (Meneveau and Sreenivasan, 1987). This model has been used to demonstrate generation of multifractal fields and their basic properties of singularities (Agterberg 2001, 2007a; Cheng, 2005; Ford and Blenkinsop, 2009). Other modifications to the model, for example, a cascade model with functional redistribution rate (Agterberg, 2007b) and a cascade model with variable partition processes (Cheng, 2005), are also available. A one-dimensional de Wijs' cascade model is to be used in this paper for convenience in introducing the association between MCP and information integration processes. The de Wijs' cascade model involves the partitioning of each unit segment into two sub-segments of equal size. The amount of measure $(\mu)$ in the unit segment then can be written as $d \times \mu$ for one half and (1-d) $\times \mu$ for the other half $(0<d<1)$ so that total mass is preserved, $d \mu+(1-d) \mu=\mu$. The coefficient of dispersion, $d$, is independent of segment size. At the beginning of the process, $\mu$ for the first segment can be set equal to unity. If $d>1 / 2$, the maximum quantity of measure in small segment unit after $n$ subdivisions is $\mu=d^{n}$, and the minimum value is $\mu=(1-d)^{n}$; if $d<1 / 2$, the maximum and minimum values are switched. The general value of the measure in small segments after $n$ subdivisions can be represented as $\mu=d^{k}(1-d)^{n-k}$, where $0 \leq k \leq n$. The number of segments with this value is $\left(\begin{array}{l}k \\ n\end{array}\right)$. In a random cascade, larger and smaller values are assigned to segments using a discrete random variable. The frequency distribution of the measure converges to a multifractal (Mandelbrot, 1989).

Let $k / n=\xi$, where $\xi$ is a value with $0 \leq \xi \leq 1$; then the value of $\mu(\xi)=\left[d^{\xi}(1-d)^{1-\xi}\right]^{n}$. The number of cells with size $\varepsilon_{n}=(1 / 2)^{n}$ and $\mu(\xi)$ becomes $N\left(\varepsilon_{n}\right)=\left(\begin{array}{l}k \\ n\end{array}\right)$. Therefore, the multifractal patterns generated by this cascade process have many local maxima and minima, with singularity expressed as follows (Feder, 1988; Halsey et al., 1986)

$$
\begin{aligned}
& \alpha=-\frac{\xi \ln (d)+(1-\xi) \ln (1-d)}{\ln 2} \\
& f(\alpha)=-\frac{\xi \ln \xi+(1-\xi) \ln (1-\xi)}{\ln 2}
\end{aligned}
$$

The fractal dimension spectrum $f(\alpha)$ characterizes the distribution of measure with singularity $\alpha$. The maximum and the minimum values of $\alpha$ from Eq. (7a) are $\alpha_{\min }=-\log _{2}(1-d)$ and $\alpha_{\max }=-\log _{2}[d]$, assuming $d>1 / 2$, therefore, the general value of singularity becomes the combination of the max and min values of $\alpha, \alpha=\xi \alpha_{\max }+(1-\xi) \alpha_{\min }$. It can be seen that the range of singularity $\alpha$ is related to the choice of $d$, $\Delta \alpha_{\max }=\alpha_{\max }-\alpha_{\min }=\log _{2}[d /(1-d)]$. As the value $d$ approaches $1 / 2$, the value range of singularity is reduced. If $d=1 / 2$, then $\Delta \alpha_{\max }=0$. According to the fractal dimension function, the sets with the maximum and the minimum singularity values have dimensions $f(\alpha(0))=f(\alpha(1))=0$ and the areas with $\alpha(1 / 2)=-1 / 2 \log _{2}[d(1-d)]$ have dimension $f(\alpha(1 / 2))=1$. It should be kept in mind that the relations (7a) and (7b) hold for $p$-model, and for other types of models the singularity range can be unbounded.

\section{Information integration processes for mapping mineral potential}

Identifying target areas favorable for undiscovered mineral deposits is essential not only for estimating the total mineral resource potential in the study area but also for exploration for new mineral deposits. Various techniques and models have been developed for facilitating the process of predictive mapping for undiscovered mineral deposits (e.g., Bonham-Carter, 1994). These methods including weights of evidence model, logistic regression, fuzzy logic, and fuzzy weights of evidence are commonly used to create predictive maps by combining multiple geoscience layers of information (i.e., evidence) associated with the occurrence of mineral deposits (e.g., Bonham-Carter, 1994; Cheng and Agterberg, 1999). The layers of spatial evidence are often derived from multiple data sources, at multiple scales and in various formats. Integrating these types of geoinformation can reduce the spatial extent of target areas and update information about the sought-after mineral deposits. The weights of evidence method is a spatial decision support model integrating map layers of information for prediction of spatial events (often but not limited to point events) (Bonham-Carter et al., 1988). 


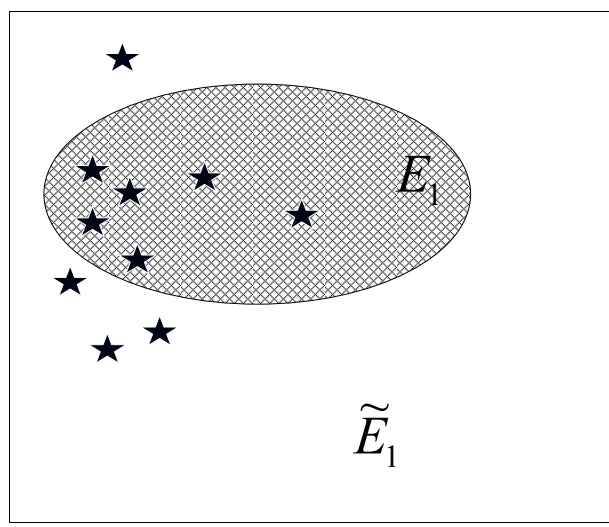

Fig. 1. Schematic diagram showing location of mineral deposits (D) shown as stars are highly associated with igneous batholith $E_{1}$. Relatively fewer mineral deposits are located outside of batholith labeled as $\tilde{E}_{1}$.

Successive overlay of evidential layers progressively partitions the study area into smaller sub-areas with updated posterior probability of containing points per unit area. In this paper it will be shown that the process of integrating layers of information has similarities with the multiplicative cascade process introduced previously. For convenience without loss of generality, we will use binary evidential layers as an example to illustrate this relationship. We need to define some notations as follows.

Let $T$ represent a study area (a 2-D set); $\left\{E_{i}, \tilde{E}_{i}\right\}(i=$ $1,2, \ldots, n)$ represent series of maps of mutually exclusive binary patterns, $E_{i}$ and $\tilde{E}_{i}, E_{i} \cap \tilde{E}_{i}=\phi$, and $E_{i} \cup \tilde{E}_{i}=T$, where " $\cap$ " and " $\cup$ " stand for intersection and union, respectively. From now on an intersection of sets appears like a product of them. Superimposing these binary patterns divides the study area $T$ into smaller sub-areas. For example, four possible intersections: $E_{i} E_{j}, E_{i} \tilde{E}_{j}, \tilde{E}_{i} E_{j}, \tilde{E}_{i} \tilde{E}_{j}$ can be formed when two maps, $\left\{E_{i}, \tilde{E}_{i}\right\}$ and $\left\{E_{j}, \tilde{E}_{j}\right\}$ are combined. To illustrate the definitions and binary patterns and their associations with mineral deposits $(D)$, several schematic diagrams are provided in Figs. 1 to 3 to present two binary maps, igneous batholith and alteration zone, considered as controlling factors for the occurrence of hydrothermal mineral deposits $D$. Each of the two binary maps divides the study area into two sub-classes: favorable areas $(E)$ and unfavorable areas $(\tilde{E})$ for formation of mineral deposits, $E \cup \tilde{E}=T$ and $E \tilde{E}=\Phi$. In this artificial example, 10 discovered mineral deposits are labeled as dots on the map and $60 \%$ of them are located on the igneous batholith $\left(E_{1}\right)$ and $40 \%$ off the igneous batholith $\left(\widetilde{E}_{1}\right), 70 \%$ of points on alteration zone $\left(E_{2}\right)$ and $30 \%$ off alteration zone $\left(\tilde{E}_{2}\right)$. The binary patterns are defined according to certain geological features, some of them with natural boundaries such as contacts between rock formations, while other patterns could be defined as manmade features with thresholds such as geological anomalies with concentration values above a certain cutoff value.

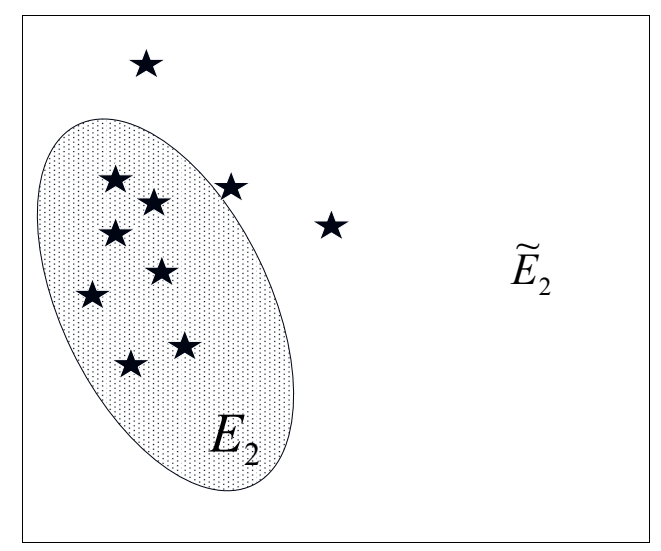

Fig. 2. Schematic diagram showing location of mineral deposits (D) shown as stars are highly associated with alteration zone labeled as $E_{2}$. Relatively fewer mineral deposits are located outside of alternation zone labeled as $\tilde{E}_{2}$.

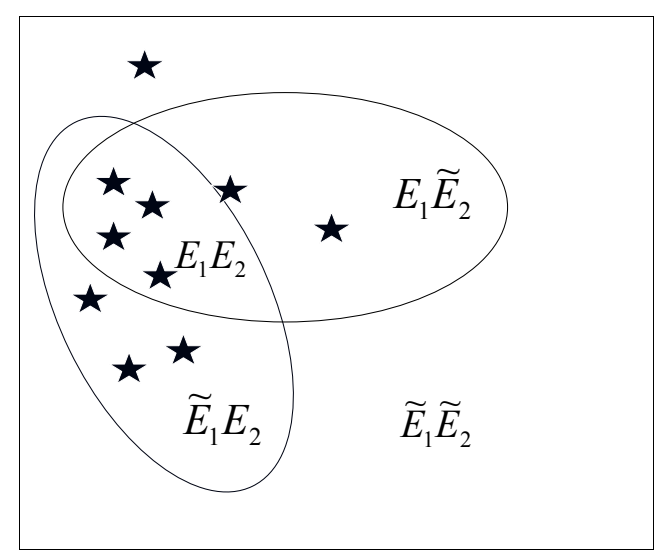

Fig. 3. Schematic diagram showing mineral deposits $(D)$ shown as stars and two binary maps partitioning the study area into four subareas labeled as $E_{1} E_{2}, E_{1} \tilde{E}_{2}, \tilde{E}_{1} E_{2}$ and $\tilde{E}_{1} \tilde{E}_{2}$, respectively.

In order to associate point events (e.g., mineral deposits in a mineral district) with these binary maps, we define probabilities and conditional probabilities as follows. Assume a small map unit is defined (for example pixel of image) so that each point of $D$ occupies only one map unit, then the number of map units occupied by mineral deposits and by map patterns $E_{i}$ and $\tilde{E}_{i}$ can be used to estimate the probabilities and conditional probabilities. For example, the prior probability, $P[D]$, of a randomly selected map unit containing a mineral deposit can be estimated by the number of map units occupied by mineral deposits, $n(D)$, divided by the total number of map units occupied by $T, n(T)$ as

$P[D]=\frac{n(D)}{n(T)}$ 
We denote prior probability of mineral deposits as $P[D]$. Similarly, we can define the probabilities of events $E_{i}, E_{j}$ and their intersections. The conditional probability of $D$ given condition of patterns $E_{i}$ and $\tilde{E}_{i}$ can be accordingly defined and denoted as $P\left[D \mid E_{i}\right]$ and $P\left[D \mid \tilde{E}_{i}\right]$, respectively. These conditional probabilities can be estimated as the ratio of the number of map units occupied both by mineral deposits and by pattern $E_{i}$ or $\tilde{E}_{i}$ over the total number of map units occupied by $E_{i}$ or $\tilde{E}_{i}$, respectively

$$
\begin{aligned}
& P\left[D \mid E_{i}\right]=\frac{n\left(D E_{i}\right)}{n\left(E_{i}\right)} \\
& P\left[D \mid \tilde{E}_{i}\right]=\frac{n\left(D E_{i}\right)}{n\left(\tilde{E}_{i}\right)}
\end{aligned}
$$

where $n(D E)$ and $n(D \tilde{E})$ represent the number of map units occupied both by mineral deposits and by pattern $E_{i}$ or $\tilde{E}_{i}$, respectively. If $P[D]$ is considered as the initial measure of mineral deposits in the entire area, each addition of an evidential layer of binary pattern $\left(E_{i}\right.$ and $\left.\tilde{E}_{i}\right)$ will produce one sub-area (favorable area) with a conditional (posterior) probability that is increased (enhanced), $P\left[D \mid E_{i}\right]>$ $P[D]$, and another sub-area (unfavorable areas) with a conditional (posterior) probability that is decreased (depleted), $P\left[D \mid \tilde{E}_{i}\right]<P[D]$.

Similarly, we can define probabilities and conditional probabilities for $D$ and any possible multiple intersections of patterns, for example,

$$
P\left[D \mid E_{1}^{*} E_{2}^{*} \ldots E_{n}^{*}\right]
$$

where $E_{1}^{*} E_{2}^{*} \ldots E_{n}^{*}\left(E_{i}^{*}=E_{i}\right.$ or $\left.\tilde{E}_{i}\right)$ is an intersection of $n$ patterns. In the following sections we will discuss properties of the process of integrating map patterns.

In order to ensure the process of integration of binary patterns can reduce the areas with unique intersection of patterns, we make the further assumption: all probabilities and conditional probabilities are positive and all patterns are conditionally independent from each other with respect to $D$,

$$
\begin{aligned}
& P[D]>0, P\left[E_{i}\right]>0, P\left[\tilde{E}_{i}\right]>0 \\
& P\left[E_{i} \mid D\right]>0, P\left[\tilde{E}_{i} \mid D\right]>0 \\
& P\left[E_{1}^{*} E_{2}^{*} \ldots E_{n}^{*} \mid D\right]=P\left[E_{1}^{*} \mid D\right] P\left[E_{2}^{*} \mid D\right] \ldots P\left[E_{n}^{*} \mid D\right]
\end{aligned}
$$

The main goal of information integration processes is to combine multiple binary map patterns to divide the entire area into smaller areas on the basis of unique intersections of patterns $P\left[E_{1}^{*} E_{2}^{*} \ldots E_{n}^{*}\right]$ and to calculate the corresponding posterior probabilities $P\left[D \mid E_{1}^{*} E_{2}^{*} \ldots E_{n}^{*}\right]$. The posterior probability map shows areas with high or low probabilities of having mineral deposits and this type of information is useful for mineral exploration. In the following sections we will investigate information integration model from the point of view of cascade processes.
From the conditional independency condition (11c) we can derive the following relation between conditional probability and the probabilities of patterns

$$
\begin{aligned}
& 2 \ln P\left[E_{1}^{*} E_{2}^{*} \ldots E_{n}^{*} \mid D\right]= \\
& \alpha\left(E_{1}^{*}\right) \ln P\left[E_{1}^{*}\right]+\alpha\left(E_{2}^{*}\right) \ln P\left[E_{2}^{*}\right]+\ldots+\alpha\left(E_{n}^{*}\right) \ln P\left[E_{n}^{*}\right] \\
& 2 \ln P\left[D \mid E_{1}^{*} E_{2}^{*} \ldots E_{n}^{*}\right]= \\
& \frac{P[D]}{P\left[E_{1}^{*} E_{2}^{*} \ldots E_{n}^{* *}\right]}\left\{\alpha\left(E_{1}^{*}\right) \ln P\left[E_{1}^{*}\right]+\alpha\left(E_{2}^{*}\right) \ln P\left[E^{*}{ }_{2}\right]+\right. \\
& \left.\ldots .+\alpha\left(E_{n}^{*}\right) \ln P\left[E_{n}^{*}\right]\right\}
\end{aligned}
$$

where each coefficient can be expressed as

$$
\begin{aligned}
& \alpha\left(E_{i}^{*}\right)=\alpha\left(E_{i}\right) \text { or } \alpha\left(\tilde{E}_{i}\right) \\
& \alpha\left(E_{i}\right)=2 \frac{\ln P\left[E_{i} \mid D\right]}{\ln P\left[E_{i}\right]} \\
& \alpha\left(\tilde{E}_{i}\right)=2 \frac{\ln P\left[\tilde{E}_{i} \mid D\right]}{\ln P\left[\tilde{E}_{i}\right]}
\end{aligned}
$$

This form (12) is a log-linear model associating the conditional probabilities $P\left[D \mid E_{1}^{*} E_{2}^{*} \ldots E_{n}^{*}\right]$ and each probability of pattern $P\left[E_{i}^{*}\right]$. Therefore, the form (12) with coefficients (13) provides a new model for calculating posterior probability. This model will be compared with weights of evidence model in Sect. 5.

\section{Information integration processes and multifractal distribution}

\subsection{Conditional independent cascade processes}

In order to show the similarity between the information integration model discussed in Sect. 3 and generalized binomial cascade processes, the simple one-dimensional de Wijs's multiplicative cascade processes introduced in Sect. 2 will be compared with each other. The de Wijs's cascade processes involve binary partitioning of each unit segment into two equal segments in each generation. For random partitioning, knowledge of the details of the partition is not required, so we can use probability notation to represent the processes. Comparing the notations used for information integration with those introduced in de Wijs' model, we can define a measure as the proportion of map units containing $D$ as well as measures for the proportions of map units on $D$ and the various patterns $E_{i}$ using joint probabilities. Suppose initial measure in the entire area is defined as

$\mu=\frac{n(D)}{n(T)}=P[D]$

and measures on each pair of subareas for patterns $E_{i}$ and $\tilde{E}_{i}$ after a partition as

$$
\begin{aligned}
& \mu\left[E_{i}\right]=P\left[D E_{i}\right] \\
& \mu\left[\tilde{E}_{i}\right]=P\left[D \tilde{E}_{i}\right]
\end{aligned}
$$


Similarly, we consider the ratio of the linear size of set $E^{*}$ and the size of the entire area as the measuring scale

$\varepsilon^{2}=\frac{n\left(E^{*}\right)}{n(T)}=P\left[E^{*}\right]$

The dispersion coefficient $d_{i}$ and $1-d_{i}$ as proportions of measures redistributed in $E_{i}$ and $\tilde{E}_{i}$ involved in the partition can be expressed as

$$
\begin{aligned}
& d_{i}=\frac{\mu\left[E_{i}\right]}{\mu[D]}=P\left[E_{i} \mid D\right] \\
& 1-d_{i}=\frac{\mu\left[\tilde{E}_{i}\right]}{\mu[D]}=P\left[\tilde{E}_{i} \mid D\right]
\end{aligned}
$$

According to the definitions of the probabilities of the events $P\left[E_{1}^{*} E_{2}^{*} \ldots E_{n}^{*}\right]$, the area sizes of these events (number of map units containing a specific combination of patterns) are proportional to the probabilities

$n\left(E_{1}^{*} E_{2}^{*} \ldots E_{n}^{*}\right)=n(T) P\left[E_{1}^{*} E_{2}^{*} \ldots E_{n}^{*}\right]$

where $n(T)$ is the total number of map units covering the entire study area. Due to the property that every further partition will reduce the total area into two subareas, the size of the partitioned subareas generally decreases with increase of numbers of partitions. Further the positive probability requirement and conditional independency assumption given in Eq. (11a to c) ensure the following monotonically descending relationship

$$
P\left[E_{1}^{*} E_{2}^{*} \ldots E_{k-1}^{*}\right]>P\left[E_{1}^{*} E_{2}^{*} \ldots E_{k}^{*}\right]
$$

Relation (19) must be true because, otherwise, if $P\left[E_{1}^{*} E_{2}^{*} \ldots E_{k-1}^{*}\right]=P\left[E_{1}^{*} E_{2}^{*} \ldots E_{k}\right]$ it would follow that $P\left[E_{1}^{*} E_{2}^{*} \ldots \tilde{E}_{k}\right]=P\left[E_{1}^{*} E_{2}^{*} \ldots E_{k-1}^{*}\right]-P\left[E_{1}^{*} E_{2}^{*} \ldots E_{k}\right]=0$, and, therefore, $P\left[E_{1}^{*} E_{2}^{*} \ldots \tilde{E}_{k} \mid D\right]=0$, according to the conditional independency assumption (11c) it must exist one evidence so that $P\left[E_{i}^{*} \mid D\right]=0$, which would violate the condition (11a to $b$ ). The relation (19) indicates that the sizes of the subareas defined by the intersection of patterns $E_{1}^{*} E_{2}^{*} \ldots E_{n}^{*}$ monotonically decrease with increasing number of partitions. Keep in mind that without conditional independency assumption among $E_{1}^{*} E_{2}^{*} \ldots E_{n}^{*}$ (Eq. 11c) the strict monotonic relation (19) may not be held and some subareas delineated by intersection of sets may not approach to zero. In comparison with the relations (1) and (2), the end product may show multifractal distribution with singularities expressed as

$$
\begin{aligned}
& P\left[D E_{k_{1}}^{*} E_{k_{2}}^{*} \ldots E_{k_{n}}^{*}\right] \propto P\left[E_{k_{1}}^{*} E_{k_{2}}^{*} \ldots E_{k_{n}}^{*}\right]^{\alpha / 2} \\
& P\left[D \mid E_{k_{1}}^{*} E_{k_{2}}^{*} \ldots E_{k_{n}}^{*}\right] \propto P\left[E_{k_{1}}^{*} E_{k_{2}}^{*} \ldots E_{k_{n}}^{*}\right]^{\alpha / 2-1}
\end{aligned}
$$

The form (20) may be not valid in general but its validity in some special cases will be proved as to be discussed in the next section. If the form (20) holds true, then the singularity index value $\alpha$ determines the rate of change of the posterior probability when the number of partitions is increased with reduction of areas proportioned to $P\left[E_{1}^{*} E_{2}^{*} \ldots E_{n}^{*}\right]$ in three situations:
1. if and only if $\alpha>2$, then $P\left[D \mid E_{1}^{*} E_{2}^{*} \ldots E_{n}^{*}\right]$ decreases;

2. if and only if $\alpha<2$, then $P\left[D \mid E_{1}^{*} E_{2}^{*} \ldots E_{n}^{*}\right]$ increases;

3. if and only if $\alpha=2$, then $P\left[D \mid E_{1}^{*} E_{2}^{*} \ldots E_{n}^{*}\right]$ is unchanged.

These three properties imply that the posterior probability increases when singularity index $\alpha<2$, decreases when $\alpha>2$ and remains unchanged when $\alpha=2$.

\subsection{Independent and constant cascade processes}

To demonstrate the analogies between the de Wijs model and the pattern partitions, we will make following assumptions in addition to the conditional independency assumption (11c):

(1) All partitions are independent from each other

$P\left[E_{1}^{*} E_{2}^{*} \ldots E_{n}^{*}\right]=P\left[E_{1}^{*}\right] P\left[E_{2}^{*}\right] \ldots P\left[E_{n}^{*}\right]$

(2) All binary events have the same probability and the same conditional probability

$$
\begin{aligned}
& P\left[E_{i}\right]=P[E], P\left[\tilde{E}_{i}\right]=P[\tilde{E}] \\
& d=P\left[E_{i} \mid D\right]=P[E \mid D], 1-d=P\left[\tilde{E}_{i} \mid D\right]=P[\tilde{E} \mid D]
\end{aligned}
$$

Now we can derive the probability of $D$ on the subarea with $k$ occurrences of $E$ and $n-k$ times $\tilde{E}$ when combining n binary patterns $E_{1}^{*} E_{2}^{*} \ldots E_{n}^{*}$ as

$$
\begin{aligned}
& P\left[D E^{k} \tilde{E}^{n-k}\right]=P[D] P\left[E^{k} \tilde{E}^{n-k} \mid D\right]= \\
& P[D](P[E \mid D])^{k}(P[\tilde{E} \mid D])^{n-k}=P[D] d^{k}(1-d)^{n-k}
\end{aligned}
$$

Accordingly, the probability of $P\left[E^{k} \tilde{E}^{n-k}\right]$ can be written as

$P\left[E^{k} \tilde{E}^{n-k}\right]=(P[E])^{k}(P[\tilde{E}])^{n-k}$

Therefore, the quantity of $D$ on subareas defined with $k$ times $E$ and $n-k$ times $\tilde{E}$ can be written as $\mu=P[D] d^{k}(1-d)^{n-k}$ and the number of such subareas as $N=\left(\begin{array}{l}k \\ n\end{array}\right)$. Let $k / n=$ $\xi$ and considering the size of subareas is proportional to $P\left[E^{k} \tilde{E}^{n-k}\right]$; then

$\alpha=2 \frac{\xi \ln P[E \mid D]+(1-\xi) \ln P[\tilde{E} \mid D]}{\xi \ln P[E]+(1-\xi) \ln P[\tilde{E}]}$

$f(\alpha)=2 \frac{\xi \ln \xi+(1-\xi) \ln (1-\xi)}{\xi \ln P[E]+(1-\xi) \ln P[\tilde{E}]}$

According to relation (25a), we obtain the maximum and minimum singularities as

$\alpha(E)=2 \ln P[E \mid D] / \ln P[E]$

$\alpha(\tilde{E})=2 \ln P[\tilde{E} \mid D] / \ln P[\tilde{E}]$ 
and further the following forms

$$
\begin{aligned}
& P[E \mid D]=P[E]^{\alpha(E) / 2} \\
& P[\tilde{E} \mid D]=P[\tilde{E}]^{\alpha(\tilde{E}) / 2} \\
& P[D \mid E]=P[D] P[E]^{\alpha(E) / 2-1} \\
& P[D \mid \tilde{E}]=P[D] P[\tilde{E}]^{\alpha(\tilde{E}) / 2-1}
\end{aligned}
$$

The Eq. (27) is a special form of Eq. (20). Therefore, from Eqs. (25a) and (26) it follows that the general value of singularity is a linear combination of the values $\alpha(E)$ and $\alpha(\tilde{E})$. If we further assume $P[E]=P[\tilde{E}]=0.5$, then relations (25a) and (25b) become the same relations as shown in Eq. (7a) and (7b). In this special case each partition divides the previously divided subareas into two equal subareas and the dispersion coefficient $d$ is independent of the partitioning.

\section{Weights of evidence model for information integration in predictive mapping}

Several methods have been developed for combining multiple layers of evidence $\left(E_{1}^{*} E_{2}^{*} \ldots E_{n}^{*}\right)$ to map the posterior probability $P\left[D \mid E_{1}^{*} E_{2}^{*} \ldots E_{n}^{*}\right]$ for prediction of mineral deposits. The weights of evidence method is one of the most commonly used methods for integration of evidential layers for predictive purposes (Bonham-Carter et al., 1988; Agterberg 1989a, b; Agterberg and Bonham-Carter, 1990; Bonham-Carter, 1994). Under the assumption of conditional independency Eq. (11a to c) the weights of evidence method gives the following logistic model for calculating posterior probability

$$
P\left[D \mid E_{1}^{*} E_{2}^{*} \ldots E_{n}^{*}\right]=\frac{1}{1+e^{-\left(W_{0}+W_{E_{1}^{*}}+W_{E_{2}^{*}}+\ldots+W_{E_{n}^{*}}\right)}}
$$

where $W_{0}$ and $W_{E *}$ are weights as shown below

$$
\begin{aligned}
& W_{o}=\ln \left(\frac{P[D]}{P[\tilde{D}]}\right) \\
& W_{E_{i}}=\ln \left(\frac{P\left[E_{i} \mid D\right]}{P\left[E_{i} \mid \tilde{D}\right]}\right), W_{\tilde{E}_{i}}=\ln \left(\frac{P\left[\tilde{E}_{i} \mid D\right]}{P\left[\tilde{E}_{i} \mid \tilde{D}\right]}\right)
\end{aligned}
$$

In order to compare the logistic model (28) and the new model (12), we will show the association between the weights $W_{E}$ and $W_{\tilde{E}}$ shown in Eq. (29) and the singularity indices $(E)$ and $\alpha(\tilde{E})$ in Eq. (13).

We can see from their definitions that the main differences between $W_{E}, W_{\tilde{E}}$ and $\alpha(E), \alpha(\tilde{E})$ are that the former involve $P[E \mid \tilde{D}], P[\tilde{E} \mid \tilde{D}]$ but the latter involve only $P[E]$ and $P[\tilde{E}]$. Since the latter do not involve any associations of patterns and the complementary event $\tilde{D}$, it is relatively easier to calculate the singularity indices $\alpha(E)$ and $\alpha(\tilde{E})$ than the weights $W_{E}$ and $W_{\tilde{E}}$. Otherwise, these two types of indices carry the same type of information about the association of patterns $E, \tilde{E}$ and event $D$ as can be concluded from the following properties:
(1) $W_{E}=0 \leftrightarrow \alpha(E)=2, W_{\tilde{E}}=0 \leftrightarrow \alpha(\tilde{E})=2$;
(2) $W_{E}>0 \leftrightarrow \alpha(E)<2, W_{\tilde{E}}>0 \leftrightarrow \alpha(\tilde{E})<2$;
(3) $W_{E}<0 \leftrightarrow \alpha(E)>2, W_{\tilde{E}}<0 \leftrightarrow \alpha(\tilde{E})>2$;

We just need to prove the first property (1) and the other two properties can be proved similarly. For example, if $W_{E}=0$, then $P[E \mid D]=P[E \mid \tilde{D}]$, which leads to $P[E \mid D]=$ $P[E]$ so that $\alpha(E)=2$. On the other hand, if $\alpha(E)=$ 2, then $P[E \mid D]=P[E]$ so that $P[E D]=P[E] P[D]$ and further, $P[E \tilde{D}]=P[E]-P[E D]=P[E]-P[E] P[D]=$ $P[E] P[\tilde{D}]$, therefore, $P[E \mid \tilde{D}]=P[E]$ and $W_{E}=0$. This proves the first property (1) implying that $D$ and $E$ are independent. The other two properties correspond to positive correlation between $D$ and $E$, and $P[E \mid D]>P[E \mid \tilde{D}]$ or $P[E \mid D]>P[E]$ and negative correlation between $D$ and $E$, $P[E \mid D]<P[E \mid \tilde{D}]$ or $P[E \mid D]<P[E]$, respectively.

\section{Application of information integration processes in mineral resources assessment}

\subsection{Study area and data}

To demonstrate the application of the new model (13), a case study was chosen for prediction of locations for Sn mineral deposits in the Gejiu mineral district, Yunnan, southwestern China. The geology of the study area is illustrated in Fig. 4. The study area $\left(\approx 1688 \mathrm{~km}^{2}\right)$ is mostly underlain by a sequence of Paleozoic to Mesozoic sedimentary rocks (the Gejiu and other formations) and igneous rocks, including Paleozoic volcanic rocks and Mesozoic intrusive rocks. The Gejiu batholith, which is located in the center of the Gejiu district, is a key factor for Sn mineralization. The Gejiu formation is hosted by limestone with minor dolomite and is the main rock formation hosting most of the discovered Sn deposits (Fig. 4). The main faults and folds in the eastern part of the study area have $\mathrm{N}-\mathrm{S}$ and $\mathrm{E}-\mathrm{W}$ orientations, while the main faults and folds in its western parts are NESW, NW-SE and E-W trending. The intersections of three groups of fault systems are diagrammed in Fig. 5. These fault systems control the general configuration of the mineralization and distribution of ore bodies in this area. The main trend of the mineralization in the eastern area is in the NNE-SSW orientation; however, the ore fields are concentrated along the intersections of NNE-SSW and E-W faults. The mineral assemblages associated with the mineralization include pyrrhotite, pyrite, cassiterite, galena, sphalerite and arsenopyrite. The Sn ores also contain relatively high concentrations of the trace elements $\mathrm{Pb}, \mathrm{Zn}, \mathrm{Cu}, \mathrm{As}, \mathrm{Sb}, \mathrm{Bi}, \mathrm{Au}$, and Ag. More comprehensive descriptions of the geology, geochemistry and mineralogy of the area can be found in Qing et al. (2004) and Yu et al. (1988). 


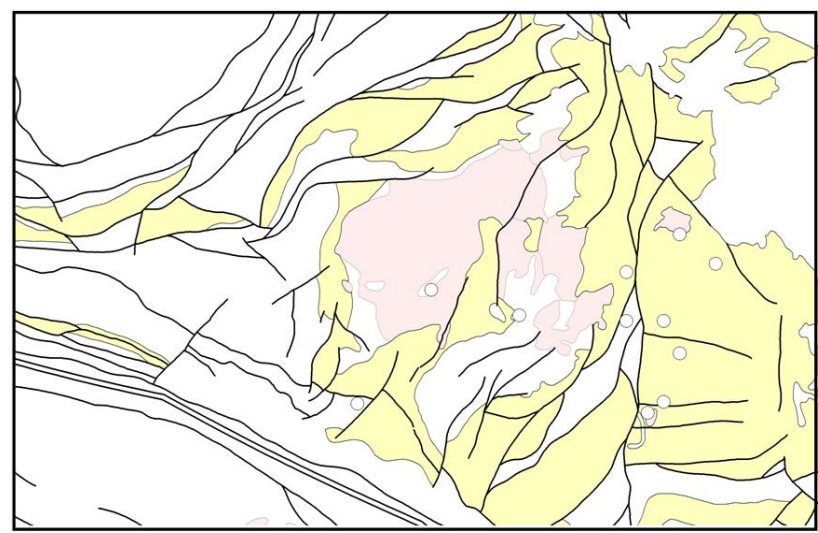

Fig. 4. Simplified geology of Gejiu mineral district. Pink polygons distributed in the center of the study area stand for Gejiu Batholith of felsic intrusions, yellow polygons for Gejiu formation of limestone, black lines represent faults, white areas for other sedimentary rocks, and dots for Sn mineral deposits.

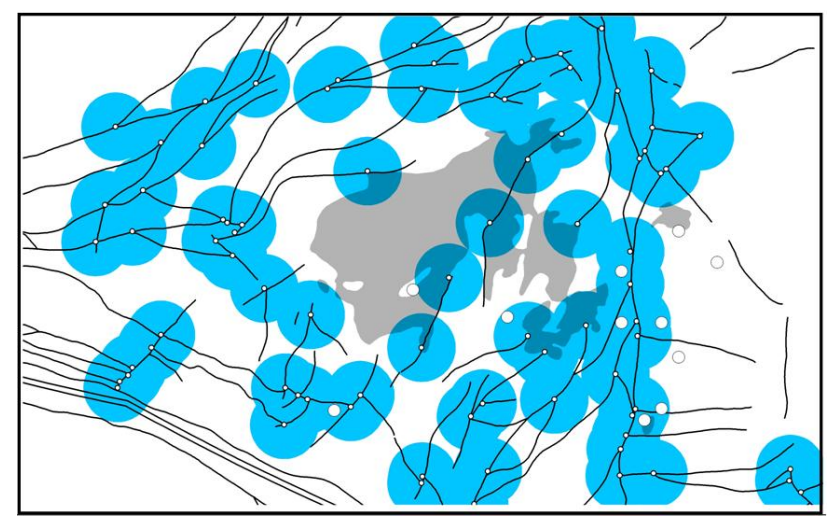

Fig. 5. Faults systems. Black lines represent faults. Blue circles stand for $6 \mathrm{~km}$ buffer zones around intersections of three fault systems.

In the author's previous studies, four factors related to mineralization were identified according to a hydrothermal mineral deposit genesis model; accordingly, four binary layers were constructed using various GIS and spatial analysis techniques. These four layers are: (1) Gejiu formation of carbonates (Gf) (Fig. 4); (2) the $6 \mathrm{~km}$ buffer zone around the intersections of three groups of faults (Bf) (Fig. 5); (3) local geochemical anomalies (La) delineated from concentration values of the elements $\mathrm{Sn}, \mathrm{As}, \mathrm{Zn}, \mathrm{Pb}, \mathrm{Cu}$ and $\mathrm{Cd}$ in stream sediment samples (Fig. 6); and (4) regional geochemical anomalies ( $\mathrm{Ra}$ ) delineated from values of $\mathrm{Sn}, \mathrm{As}, \mathrm{Zn}$, $\mathrm{Pb}, \mathrm{Cu}$ and $\mathrm{Cd}$ in stream sediment samples (Fig. 7). More detailed information about the definition of the four binary layers can be found in Cheng et al. (2009). This paper will use these four binary layers to calculate the posterior probability map according to relation (12) and to demonstrate the processes of combining layers and the singularities of the created posterior probability map.

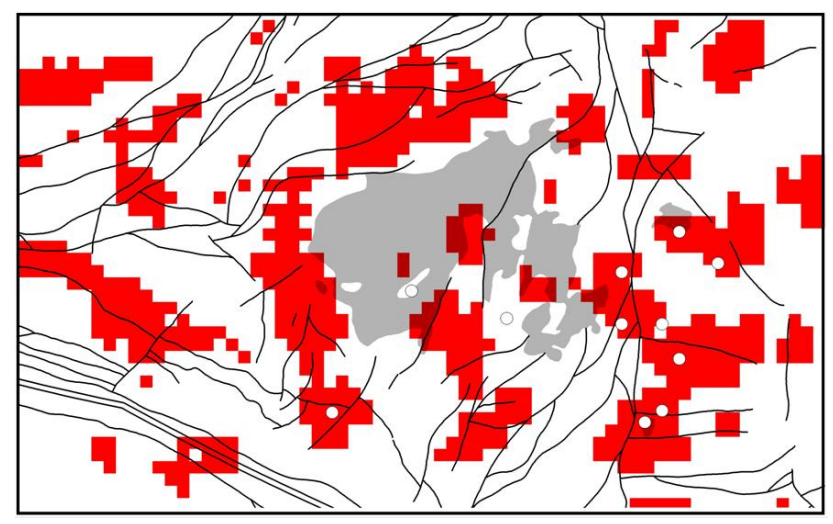

Fig. 6. Local geochemical anomalies extracted from concentration valelements $\mathrm{Sn}, \mathrm{As}, \mathrm{Cu}, \mathrm{Pb}, \mathrm{Zn}$ and $\mathrm{Cd}$. First singularity index was cathe concentration valuesues of lculated from of each element and these singularity maps were combined by means of principal component analysis. The patterns in red are the binary patterns created from the score on the first component. Details can be found in Cheng et al. (2009).

\subsection{Results}

Each information layer to be combined (binary patterns of which are shown in Figs. 4 through 7) divides the study area into two subclasses of reduced area that represent favorable and unfavorable regions for predicting mineral deposits. If we define a square of size $1 \times 1 \mathrm{~km}^{2}$ as the measuring unit, the total study area occupies about 1688 units. There are a total of 11 units occupied by Sn mineral deposits, from which the prior probability of a randomly chosen square from the area containing mineral deposits can be estimated as $P[D]=$ 0.0065 . Similarly, one can calculate the number of units occupied by binary patterns and the number occupied by both binary patterns and mineral deposits. Table 1 gives the results obtained from the four binary maps Gf, Bf, La and Ra. The second and third columns in Table 1 show the areas (number of units) of the favorable patterns of each binary map and the numbers of mineral deposits occurring in each of the patterns (\# points on pattern). From these two columns, one can calculate the probability of patterns for each of the four binary maps; for example, probability of Geijiu formation $P[\mathrm{Gf}]=$ $747 / 1688=0.44$ and $P[\tilde{\mathrm{G} f}]=1-P[\mathrm{Gf}]=0.56$, representing the probabilities that patterns exist on the Gejiu formation and the complementary event (on other rock types). One can also calculate the probability that a unit area of a given binary pattern contains mineral deposits, for example, $d=P[\mathrm{Gf} \mid D]=7 / 11=0.64$ and $1-d=P[\tilde{\mathrm{G}} \mid D]=0.36$; these values represent, respectively, the conditional probabilities of mineral deposits on and off the pattern Gf. From these probabilities and conditional probabilities, one can calculate the singularity indices $\alpha(E)$ and $\alpha(\tilde{E})$ for each binary pattern; for example, $\alpha(\mathrm{Gf})=2 \ln P[\mathrm{Gf} \mid D] / \ln P[\mathrm{Gf}]=1.11$ and $\alpha(\tilde{\mathrm{G}} \mathrm{f})=2 \ln P[\tilde{\mathrm{G}} \mathrm{f} \mid D] / \ln P[\tilde{\mathrm{G}}]=3.46$. The statistics for 
Table 1. Statistics obtained from each of the four layers of binary maps.

\begin{tabular}{lrrrrrrrrrrrrr}
\hline & Area & $\begin{array}{r}\# \\
\text { points }\end{array}$ & $W_{E}$ & $s\left(W_{E}\right)$ & $W_{\tilde{E}}$ & $s\left(W_{\tilde{E}}\right)$ & & $s(C)$ & t-value & $\alpha$ & $\alpha(\tilde{E})$ & $\begin{array}{r}\Delta \alpha \\
\alpha_{\text {max }}-\alpha_{\text {min }}\end{array}$ \\
\hline Rock Type (Gf) & 747 & 7 & 0.35 & 0.38 & -0.40 & 0.49 & 0.75 & 0.63 & 1.2 & 1.11 & 3.46 \\
Dist. to Inter. (Bf) & 515 & 6.4 & 0.66 & 0.40 & -0.52 & 0.47 & 1.18 & 0.61 & 1.92 & 0.91 & 4.79 & 2.35 \\
Geochem. I (La) & 188 & 8 & 1.91 & 0.36 & -1.19 & 0.58 & 3.10 & 0.68 & 4.54 & 0.29 & 22.01 & 21.72 \\
Geochem. II (Ra) & 309 & 6 & 1.12 & 0.41 & -0.61 & 0.45 & 1.73 & 0.61 & 2.84 & 0.71 & 7.8 & 7.09 \\
\hline
\end{tabular}

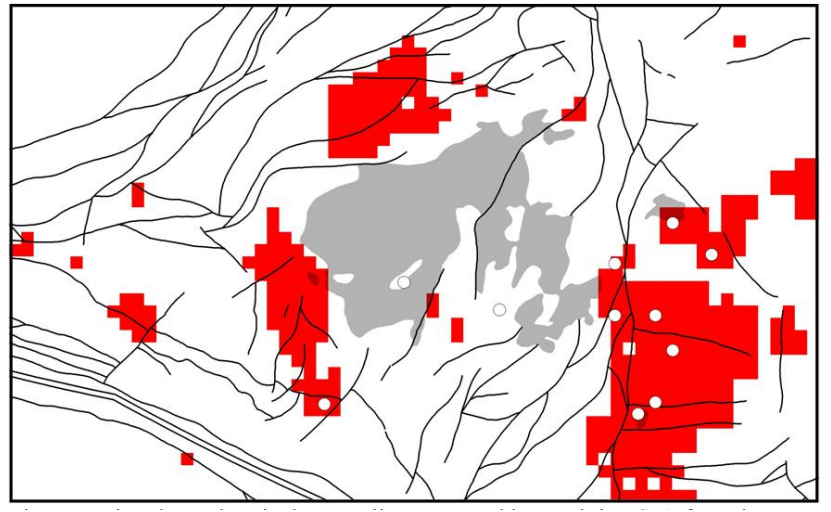

Fig. 7. Regional geochemical anomalies extracted by applying S-A filtering to the scores calculated from the first principal component fractal derived from logarithmic transformed concentration values of elements $\mathrm{Sn}, \mathrm{As}, \mathrm{Cu}, \mathrm{Pb}, \mathrm{Zn}$ and $\mathrm{Cd}$. The patterns in red are the binary patterns created from the decomposed patterns by means of S-A method. Details can be found in Cheng et al. (2009).

other binary patterns are similarly calculated and are shown in Table 1.

The other values shown in Table 1 are statistics used in the weights of evidence method. Value of $s(W)$ stands for standard deviation of $W$ and t-value is for Student's statistic value of the contrast of weights $C=W_{E}-W_{\tilde{E}}$. The results in Table 1 show that the four binary maps are all positively correlated with $D$. Among them, the local geochemical anomalies (La) show the highest contrast of weights with t-value (Student's statistic) $t=4.54$ (for calculation of $W, s(W)$, and t-values of $C$, see Bonham-Carter, 1994). The values of singularity indices $\alpha(E)=0.29<2$ and $\alpha(\tilde{E})=22.01>2$ also imply that the singularity related to pattern La is strong. In general, the ranges of singularity $\Delta \alpha=\alpha_{\max }-\alpha_{\min }$ are all positive which implies that all four layers are positively associated with $D$. In addition, the ranks of the ranges of singularity $\Delta \alpha$ are similar to the ranks of the t-values which implies that the range of singularity can be used as a statistic measuring the degree of correlation between $D$ and the patterns. Since all four map layers are associated with the location of $D$, these four layers must be combined in order to create a posterior probability map that shows the best areas

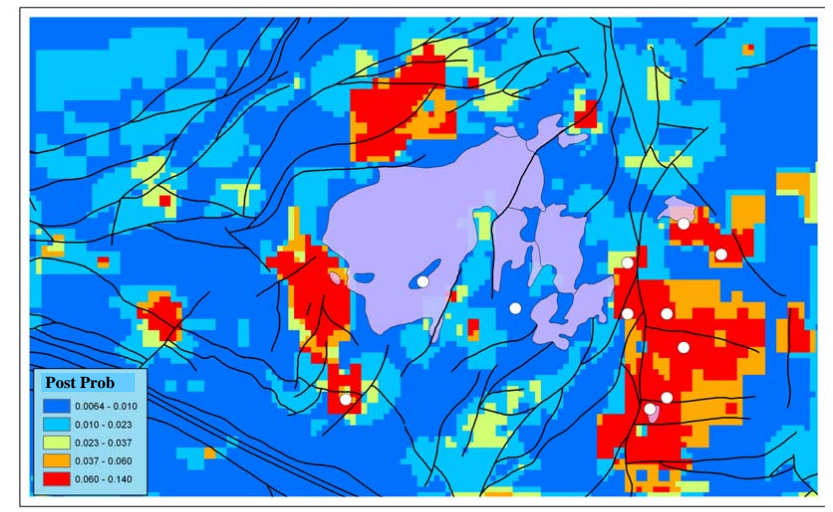

Fig. 8. Posterior probability map created by means of weights of evidence method applied to the four evidential layers in Figs. 4 to 7.

for occurrences of mineral deposits. Using model (12), four layers of binary maps were combined; the posterior probability map that emerged is shown in Fig. 8. The areas with high posterior probability values contain most of the known mineral deposits; some areas with high posterior probabilities in which mineral deposits have not yet been found can be considered target areas for further exploration.

To validate the multifractality of the posterior probability map, one can apply various multifractal modeling techniques. In this case study, since we only use four evidence layers which corresponding to four partition generation, the results are far from a fully converged multifractal field. Therefore, it is not possible to validate the multifractality of the posterior probability map in Fig. 8. In further study more evidence layers could be added to generate a more realistic multifractal posterior probability map, especially to show those areas with high posterior probability but without known mineral deposits that remain to be discovered. In order to display the frequency distribution of the posterior probability on Fig. 8, first, the value of posterior probability was transformed by logarithmic transformation (natural $\log s$ ). Next equal interval classification was applied to the transformed posterior probability values. All together, 21 classes were determined: the starting class with mid-point value of -7.1289 , the last class with 


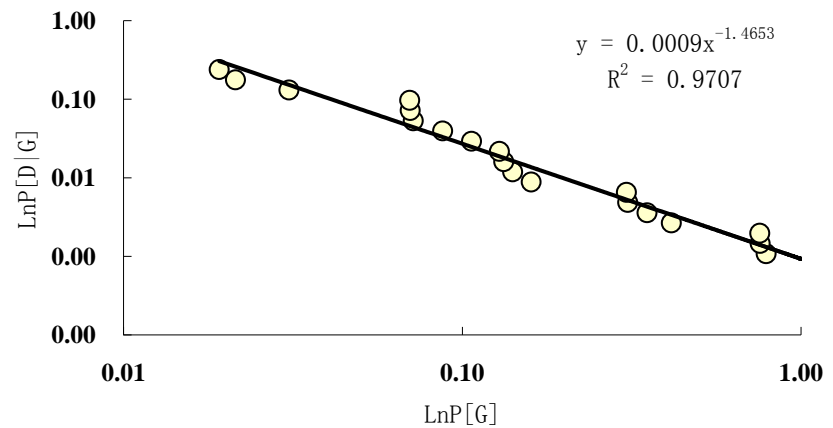

Fig. 9. Relationship between posterior probability $P[D \mid G]$ and the probability of the area $(\mathrm{G})$ with cutoff value of posterior probability.

mid-point value of -1.4354 and each class interval equal to 0.299. Cumulative frequencies of map units in each class were calculated and these values are plotted in Fig. 9. In Fig. 9 the posterior probability corresponding to the midpoint value of each class, denoted as $P[D \mid G]$ and the probability of the cumulative number of map units occupying these classes, denoted as $P[G]$, are plotted. The result generally shows a power-law relationship between the cumulative area and the mid-point class value of posterior probability, $P[D \mid G]=0.0009 P[G]^{-1.4653}$. Since the posterior probability $P[D \mid G]$ is the value at boundary of $G$, the average posterior probability in $G$ should also follow power-law relations with $P[G]$ with exponent -0.4653 which gives the singularity $\alpha / 2=0.4653$ and $\alpha=1.08<2$ implying a general enrichment of posterior probability at the peaks when the size of area $G$ decreases.

\section{Conclusions and discussion}

Multiplicative cascade processes are non-linear processes commonly observed in geoscience that can create end products that follow multifractal distribution with multi-scale singularities. Singularity is a natural property of mineralization, which involves enrichment and depletion of ore and associated elements in the Earth's crust as well as in other relevant secondary media such as tills, soils, lake and stream sediments, humus and vegetation surrounding mineral deposits. Mapping such singularities is an effective way to delineate areas favorable for occurrence of mineral deposits and estimate the likelihood of the presence of undiscovered mineral resources in a given area.

To support decision-making and to map areas for prediction of mineral deposits, multiple layers of information that are positively associated with the location of mineral deposits must often be combined. This information integration process can be considered as a type of multiplicative cascade process that updates the posterior probability by combining evidence layers. The model proposed in this paper provides an alternative log-linear relationship associating conditional probability of points and evidence layers with singularity indices as coefficients, which quantify degree of spatial correlation between binary patterns and location point events (e.g., mineral deposits). This feature is important for three reasons: first, it links the concepts and methods of multifractal modeling developed in nonlinear processes in geophysics and information integration modeling commonly employed for spatial decision support. Secondly, the newly developed log-linear model can not only be used as an alternative model for information integration but also provides relatively simpler indices in comparison with the weights of evidence method. This is because the singularity indices involved in the new model do not require calculation of probabilities and conditional probabilities related to complementary point events. In addition, as the end product of a multiplicative cascade process, the posterior probability may possess multifractality with singularities that can be further investigated for characterization of the spatial distribution of the point events. Moreover, the results and formulation developed in the current research might be useful for further study of more generalized multiplicative cascade processes. It must be kept in mind that a large number of evidential layers are usually needed to create posterior probability maps that are representative of multifractal fields. However, when the number of binary patterns increases, the dependency among the patterns and between patterns and point events becomes inevitable. Therefore, a more general model overcoming these dependency problems has to be developed.

Acknowledgements. Sincere thanks are due to the editor D. Schertzer and two anonymous reviewers for providing constructive comments which have significantly improved the paper. The author thanks Frits Agterberg for reading this manuscript and providing constructive comments. The research was financially supported by a High-Tech Research and Development Grant (2009AA06Z110) by the Ministry of Science and Technology of China, and Grants from Ministry of Education of China (No. IRT0755).

Edited by: D. Schertzer

Reviewed by: two anonymous referees

\section{References}

Agterberg, F. P.: Computer programs for exploration, Science, 245, 76-81, 1989a.

Agterberg, F. P.: Systematic approach to dealing with uncertainty of geoscience information in mineral exploration, in: Application of Computers and Operations in the mineral industry, edited by: Weiss, A., Proc. 21st APCOM Symp., Las Vegas, Nevada, Colorado Society of Mining Engineers, Littleton, 165-178, 1989b.

Agterberg, F. P.: Multifractal modeling of the sizes and grades of giant and supergiant deposits, Int. Geol. Review, 37, 1-8, 1995.

Agterberg, F. P.: Multifractal simulation of geochemical map patterns, in: Geologic modeling and simulation: Sedimentary Systems, edited by: Merriam, D. F. and Davis, J. C., Kluwer, New York, 327-346, 2001. 
Agterberg, F. P.: New applications of the model of de Wijs in regional geochemistry, Math. Geol., 39, 1-26, $2007 \mathrm{a}$.

Agterberg, F. P.: Mixtures of multiplicative cascade models in geochemistry, Nonlin. Processes Geophys., 14, 201-209, doi:10.5194/npg-14-201-2007, 2007b.

Agterberg, F. P. and Bonham-Carter, G. F.: Deriving weights of evidence from geoscience contour maps for prediction of discrete events, Proc. 22nd APCOM Symp. (Berlin, Germany), Tech. Univ. Berlin, 2, 381-396, 1990.

Blenkinsop, T. G.: The Fractal Distribution of Gold Deposits, in: Fractals and Dynamic Systems in Geoscience, edited by: Kruhl, J. H., Springer-Verlag, 247-258, 1994.

Bonham-Carter, G. F.: Geographic Information Systems for Geoscientists: Modelling with GIS, Pergamon, Oxford, 398 pp., 1994.

Bonham-Carter, G. F., Agterberg, F. P., and Wright, D. F.: Integration of geological data sets for gold exploration in Nova Scotia, Photogr. Eng. Remote Sens., 54, 1585-1592, 1988.

Carlson, C. A.: Spatial distribution of ore deposits, Geology, 19, 111-114, 1991.

Cheng, Q.: The gliding box method for multifractal modeling, Comput. Geosci., 25, 1073-1079, 1999.

Cheng, Q.: Fractal and multifractal modeling of hydrothermal mineral deposit spectrum: application to gold deposits in the Abitibi Area, Canada. J. China Univ. of Geosci., 14, 199-206, 2003.

Cheng, Q.: Multifractal distribution of eigenvalues and eigenvectors from 2-D multiplicative cascade multifractal fields, Math. Geol., 37, 915-927, 2005.

Cheng, Q.: Mapping singularities with stream sediment geochemical data for prediction of undiscovered mineral deposits in Gejiu, Yunnan Province, China, Ore Geol. Reviews, 32, 314324, 2007a.

Cheng, Q.: Multifractal imaging filtering and decomposition methods in space, Fourier frequency, and eigen domains, Nonlin. Processes Geophys., 14, 293-303, doi:10.5194/npg-14-293-2007, 2007b.

Cheng, Q.: Non-linear theory and power-law models for information integration and mineral resources quantitative assessments, Math. Geosci., 40, 503-532, 2008.

Cheng, Q. and Agterberg, F. P.: Multifractal modeling and spatial statistics, Math. Geol., 28, 1-16, 1996.

Cheng, Q. and Agterberg, F. P.: Fuzzy weights of evidence method and its application in mineral potential mapping, Nat. Res. Res., 8, 27-35. 1999.

Cheng, Q. and Agterberg, F. P.: Singularity analysis of ore-mineral and toxic trace elements in stream sediments, Comput. Geosci., 35, 234-244, 2009.

Cheng, Q., Bonham Carter, G. F., Agterberg, F. P., and Wright, D. F.: Fractal modeling in the geosciences and implementation with GIS, in: Proc of the 6th Canadian Conference on GIS, Ottawa, June 6, 10, 1, 565-577, 1994a.

Cheng, Q., Agterberg, F. P., and Ballantyne, S. B.: The separation of geochemical anomalies from background by fractal methods, J. Explor. Geochem., 51, 109-130, 1994b.

Cheng, Q., Zhao, P., Zhang, S., Xia, Q., Chen, Z., Chen, J., Xu, D., and Wang, W.: Application of singularity in mineral deposit prediction in Gejiu district: Information integration and delineation of target areas, Earth Science, 34, 243-252, 2009 (in Chinese with English abstract). de Wijs, H. J.: Statistics of ore distribution, part I, Geologie Mijnbouw, 13, 365-375, 1951.

Feder, J.: Fractals, Plenum Press, New York, 283 pp., 1988.

Ford, A. and Blenkinsop, T. G.: An expanded de Wijs model for multifractal analysis of mineral production data, Mineral Deposita, 44, 233-240, 2009.

Frisch, U., Sulem, P. L., and Nelkin, M.: A simple dynamical model of intermittency in fully develop turbulence, J. Fluid Mech., 87, 719-724, 1978.

Halsey, T. C., Jensen, M. H., Kadanoff, L. P., Procaccia, I., and Shraiman, B.: Fractal measures and their singularities: the characterization of strange sets, Phys. Rev. A, 33, 1141-1151, 1986.

Hronsky, J. M. A: Self-organized systems and ore formation: the key to spatially-predictive targeting?, Proceedings of the Tenth Biennial SGA Meeting, Townsville 2009, 19-21, 2009.

Lovejoy, S. and Schertzer, D.: Scaling and multifractal fields in the solid earth and topography, Nonlin. Processes Geophys., 14, 465-502, doi:10.5194/npg-14-465-2007, 2007.

Malamud, B. D., Turcotte, D. L., and Barton, C. C.: The 1993 Mississippi river flood: a one hundred or a one thousand year event?, Environ. Eng. Geosci., II, 479-486, 1996.

Malamud, B. D., Turcotte, D. L., Guzzetti, F., and Reichenbach, P.: Landslide inventories and their statistical properties, Earth Surf Proc. Land., 29, 687-711, 2004.

Mandelbrot, B. B.: Multifractal measures, especially for the geophysicist, Pure Appl. Geophys., 131, 5-42, 1989.

Meneveau, C. and Sreenivasan, K. R.: Simple multifractal cascade model for fully developed turbulence, Phys. Rev. Lett., 59, 1424 1427, 1987.

Qing, D., Tan, S., and Fan, Z.: Geotectonic evolution and tinpolymetallic metallogenesis in Gejiu-Dachang area, J. Mineral., 24, 118-123, 2004 (in Chinese with English Abstract).

Raines, G: Are fractal dimensions of the spatial distribution of mineral deposits meaningful?, Nat. Resour. Res., 17, 87-97, 2008.

Schertzer, D. and Lovejoy, S.: Turbulence and chaotic phenomena in fluids, edited by: Tatsumi, T., North-Holland, 505-508, 1984.

Schertzer, D. and Lovejoy, S.: The dimension and intermittency of atmospheric dynamics - Multifractal cascade dynamics and turbulent intermittency, in: Turbulent Shear Flow, edited by: Launder, B., Springer-Verlag, New York, 7-33, 1985.

Schertzer, D. and Lovejoy, S.: Physical modeling and analysis of rain and clouds by anisotropic scaling of multiplicative processes, J. Geophys. Res., 92, 9693-9714, 1987.

Schertzer, D., Lovejoy, S., Schmitt, F., Chigirinskaya, Y., and Marsan, D.: Multifractal cascade dynamics and turbulent intermittency, Fractals, 5, 427-471, 1997.

Sornette, D.: Critical Phenomena in Natural Sciences: Chaos, Fractals, Selforganization and Disorder, 2nd Edn., Springer, New York, 2004.

Turcotte, D. L.: Fractals and Chaos in Geology and Geophysics, 2nd Edn., Cambridge University Press, 1997.

Turcotte, D. L.: Fractals in petrology, Lithos, 65, 261-271, 2002.

Veneziano, D.: Multifractality of rainfall and scaling of intensityduration-frequency curves, Water Resour. Res., 38, 1-12, 2002.

Xie, S., Cheng, Q., Chen, G., Chen, Z., and Bao, Z.: Application of local singularity in prospecting potential oil/gas Targets, Nonlin. Processes Geophys., 14, 285-292, doi:10.5194/npg-14-2852007, 2007. 
Yaglom, A. M.: The influence of the fluctuation in energy dissipation on the shape of turbulent characteristics in the inertial interval, Sov. Phys. Dokl. (Engl. Transl), 2, 26-30, 1966.
Yu, C., Tang, Y., and Shi, P.: Dynamic system of Gejiu polymetallic mineralization processes, China University of Geosciences Press, Wuhan, 394 pp., 1988 (in Chinese with English Abstract). 